\title{
The Design of Intelligent Multi-function Door For College Dormitory
}

\author{
Rui Wei, Ming Tang \\ School of Intelligence Science and Information Engineering \\ Xi'an Peihua University, Xi'an, China \\ 107285984@qq.com,81408240@qq.com
}

Keywords: Multi-function; Intelligent door; Fingerprint lock; Infrared sensor

\begin{abstract}
This paper describes the design of the intelligent multi-function door. First, the overall structure of the door is designed. In this way, the detail design process is illustrated in the design of the thermal power discharge infrared sensor, the design of the fingerprint lock, the design of the server network, the design of the entrance guard module and the development of the android client. Through the technical means, the door industry can be realized, which can give an alarm automatically, monitor overall, and have comprehensive security function, and it can break through the simple "control door with lock", anti-theft idea of the traditional anti-theft door. This can make the thief difficult to attack, alarming automatically after the thief attack, and make the thief no place to hide, escape hastily and fearfully.
\end{abstract}

\section{Background}

According to the 2016 national economy and survey data released by the national bureau of statistics, there are 26 million 253 thousand college students in the whole country. In addition, the safety awareness of college students is relatively weak. The frequent occurrence of fire and theft incidents not only brings property loss and safety threat to students themselves, but also seriously affects the quality of running a colleges in universities.

Fire should be the primary problem for the dormitory safety. Students use high-power electrical appliances illegally, such as heater, hair dryer, electric kettle, pulling wires privately, connecting socket and so on. Once the electrical circuit is short, power cutting is slight, and the fire is serious. Problems caused by alcohol furnace and smoking. Alcohol furnace and smoking all need to use lighters or matches, so there are so many accidents caused by open fire.

The main reason is that many students lack the safety awareness. They leave but the door is unlocked, and lay the wallet, mobile phone and other valuables everywhere, which give the perpetrator a chance to steal.

The dormitory intelligent alarm and open door system helps college students to monitor and understand the situation in their dormitory in real time when they go out, so that students can respond to the dormitory problems in the shortest time and protect the financial security of the dormitory effectively. It provides a guarantee for the safety of College Students' dormitories; In addition, the use of fingerprint unlocking system is more convenient for college students to enter dormitories. It can ensure that college students can still enter the student dormitories without taking keys or key missing, rather than being locked out. 


\section{Main Technologies and Functions}

\section{(1) Automatic Alarm}

It uses fire detector and pyroelectric sensor to realize real-time alarm through the GSM alarm when indoor fire or strangers enter, and sends the image or video to the dormitory personnel's mobile phone, timely notifying, to let students make correct and timely treatment of the situation in the house.

\section{(2) Fingerprint Unlock}

It uses an automated fingerprint recognition mechanism to collect, analysis, and contrast of a living fingerprint automatically through an elaborate array of electronic parts and mechanical parts. And it can identify the fingerprint of the door opener automatically, quickly and accurately. If the fingerprint is correct, the door will be open.

\section{(3) Automatic Shutdown}

Using the door closer, which can be released after the door is opened, the door will be closed automatically, Similar to the function of the spring door, it can be ensured that the door is closed to the initial position accurately and in time after the door is opened. If the door is opened for more than 10 seconds without being gripped, the alarm sounds, and for more than 15 seconds it will notify the dormitory personnel that the door is not closed in time by the GSM alarm.

\section{(5) Remind the Student to Pull out the Key}

If there is a power failure in the dormitory, the dormitory staff can also open the door by the key. When the key is forgotten after the door is opened, the key can be connected with the door lock to make the alarm alert the dormitory staff to pull the key. In addition, there are emergency rechargeable batteries on the door.

\section{(6) The Ventilation System}

We can manually open it to allow indoor air to circulate when there is a fire in the dormitory or smell.

\section{Main Research Contents and Key Technology}

\subsection{Main Research Contents}

Through the Internet, communication network platform, it can carry on remote real-time monitoring and network information interaction. When the dormitory members are not in the dormitory during the day and have a rest during the night, it can turn on the monitor automatically.

When there are strangers or fire in the dormitory, they can alarm the dormitory members in time and remind the dormitory members, and upload the information of the dormitory to the mobile phone of the dormitory staff, so that the dormitory staff can understand the latest situation and make corresponding measures. Meanwhile, the alarm bells are issued by the alarm in the dormitory, so that the students in the surrounding dorm can make the correct response in time. Through the induction of the network signal, when the person is at a certain distance, the cell phone can make the prompt to open the door, and the dormitory staff can control the opening of the dormitory door by mobile phone.

\subsection{Key Technology}

\section{(1) The Design of Automatic Fire Alarm System}

Fire alarm system (Fig.1) automatic fire alarm system is a fire alarm system composed of trigger device, fire alarm device, fire alarm device and other auxiliary functions. At the beginning of the fire, through fire detector like warm, smoky and light-sensitive, it can turn the heat from the smoke, heat, and light radiation into electrical signals, transmitting to the fire alarm controller, and show 
where the fire occurred, recording the time of the fire. General fire automatic alarm system is connected with automatic sprinkler system, indoor and outdoor fire hydrant system, smoke control system, ventilation system, air conditioning system, fire door, fire shutter, smoke screen and other related equipment, and it issue instructions automatically or manually, starting up the corresponding device

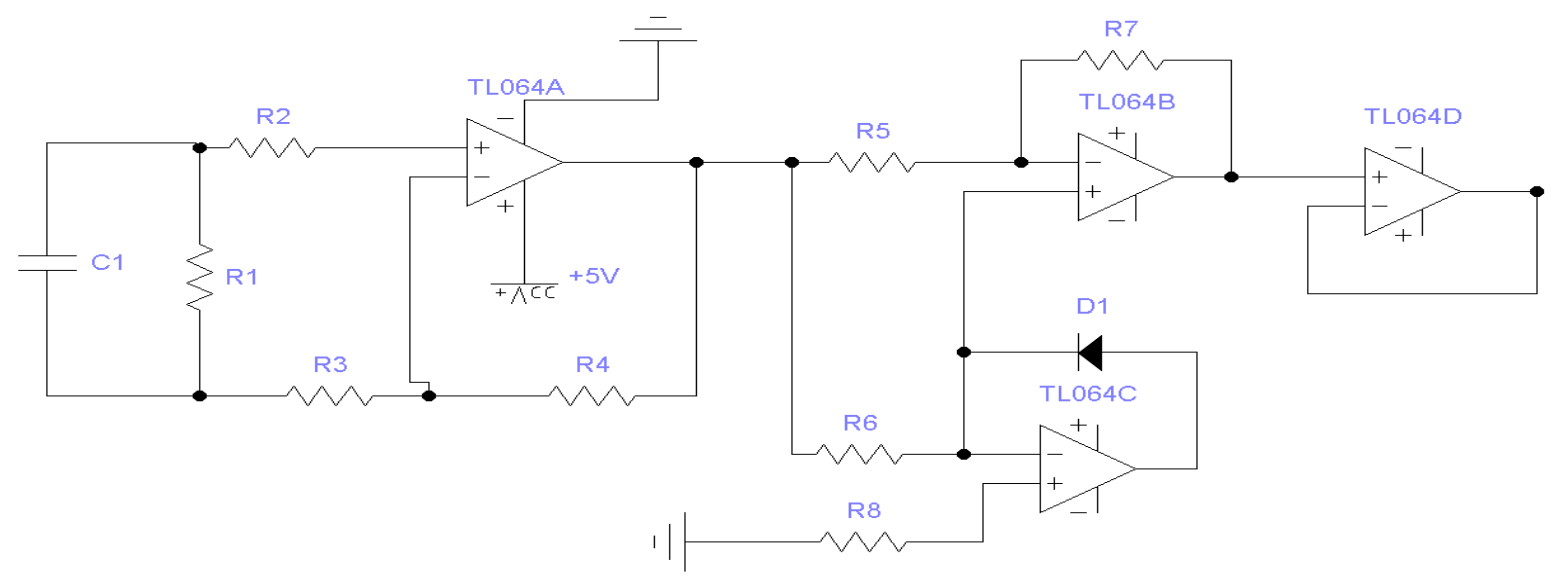

Figure 1. Fire Alarm Circuit Diagram

\section{(2) The Design of Pyroelectric Infrared Sensor}

It is mainly composed of a material with high thermoelectric coefficient, such as zirconium titan ate ceramic, lithium tantalite, triglyceride sulfate, etc., which is $2 * 1 \mathrm{~mm}$ detector. One or two detector elements are loaded into each detector, and the two detector elements are connected in reverse polarity to suppress the interference caused by their own temperature rise. The infrared radiation detected and received by the detecting element is transformed into a weak voltage signal, and it is amplified by the field effect tube mounted in the probe .In order to increase the detection sensitivity of the detector to increase the detection range, a Fresnel lens is installed in front of the detector, which is made of transparent plastic. The upper and lower parts of the lens are divided into several equal parts to make a lens with a special optical system. It works with a magnifying circuit that can amplify signals by up to 70 decibels, which can measure the movements of people within a range of 20 meters.

Fresnel lens utilizes the special optical principle of the lens to produce an alternate "blind zone" and "high sensitive area" in front of the detector to improve its detection and receiving sensitivity. When someone passes through the lens, the infrared light from the human body alternate continuously from the "blind area" to the "high sensitive area", so that the received infrared signal can be input in the form of the pulse form, which is strong and weak, thus strengthening its energy amplitude.

The infrared center wavelength of the human body radiation is 9 10--um, and the wavelength sensitivity of the detection element is almost constant in the range of 0.2 20--um. At the top of the sensor, there is a window with a filter lens, which can pass through the wavelength range of 7 10--um, which is suitable for the detection of infrared radiation of human body, and the infrared light of other wavelengths is absorbed by the filter, thus it form a kind of infrared sensor specially used for detecting human radiation.

Schematic Diagram of Pyroelectric Infrared Sensors, as shown in Fig.2. 




Shape of the sensor



Electrical schematic diagram of the sensor

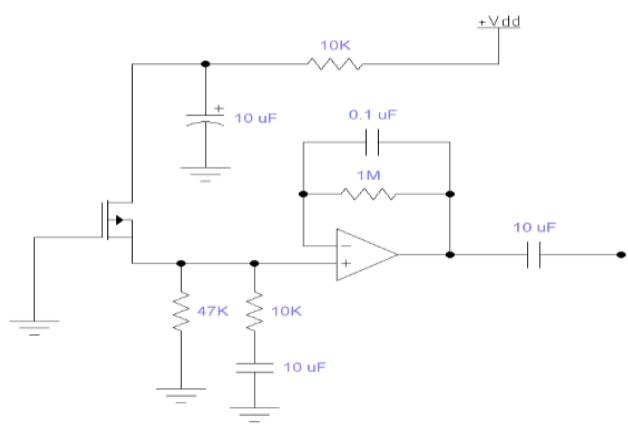

Typical application diagram of sensor

Figure 2. Schematic Diagram of Pyroelectric Infrared Sensors

\section{(3) The Design of Fingerprint Lock}

According to the uniqueness of each person's fingerprints, it is determined that the fingerprint is used as the key. The individual's fingerprints are stored in advance by pre-filing in the system. When dormitory staff have access needs, the fingerprint sensor collects the fingerprint information of the dormitory staff, and sends the fingerprint eigenvalues to the fingerprint identification chip for analysis and comparison, deciding whether the dormitory staff have access rights.

If the dormitory personnel have the necessary authority, after the verification is passed, the fingerprint identification chip outputs control signal to drive the gated DC motor to control the door. If the dormitory staff does not have the corresponding authority, validation failure will be gave after validation.

\section{(4) The Design of Automatic Shutter}

The basic components of automatic shutter include support guide, transmission gear, reset spring, one-way valve, rack plunger, throttle valve core and shell, end cover, sealing ring and connecting rod. The shell and the connecting rod play the role of fixing the closed door and connecting the door and door frames.

When opening the door, the door body drives the connecting rod movement, and makes the transmission gear turn, and drives the rack plunger to move to the right side. When the plunger is moved right, the spring is compressed and the hydraulic oil in the right cavity is pressed. The unidirectional valve ball on the left side of the plunger is opened under the action of oil pressure, and the hydraulic oil in the right cavity flows through the one-way valve into the left cavity. When the opening process is completed, due to the compression of the spring during the opening process, the elastic potential energy stored is released. The plunger is pushed to the left side to drive the transmission gear and the closed door linkage to rotate, so that the door is closed. During the spring release process, because the hydraulic oil in the left chamber of the door closer is compressed, the check valve is closed, and the hydraulic oil can only flow through the gap between the shell and the plunger, and it flows back to the right cavity through the small holes on the plunger and 2 channels with throttling core. Therefore, the hydraulic oil has the resistance to the release of the spring, that is, having the effect of the buffer by throttle, and the speed of the closing of the door is controlled. The throttle valve on the valve body can be adjusted to control the changeable closing speed of different travel sections.

\section{(5) The Design of Single Chip Microcomputer System}

Single chip microcomputer system is designed by users to achieve control applications, and is a computer application system which are formed around the single chip microcomputer's chip, All the instructions that the single chip microcomputer can carry out are its instruction system, and 
different kinds of single chip microcomputer has different instruction system . In order to make SCM can automatically complete a particular task, we must take the problem to be solved into a series of instructions (these instructions must be identified and implemented by selected single chip microcomputer), and the collection of this series of instructions is called a program, and the program needs to be stored in the parts with the storage function -- storage device in advance. The storage device is formed with many storage units (the smallest storage unit), just like a large building with many rooms, and the instructions are stored in these units. The instructions in the units are taken out and executed just like every room in a large building is assigned to a unique room number. Each storage unit must also be assigned to a unique address number, which is known as the address of the storage unit, Once know the address of the storage unit, the storage unit can be found, and the instructions stored in the storage unit can be removed and then executed.

\section{(6) Upload Alarm Technology Based on 2G Network Information}

The GSM multimedia message alarm system is composed of an infrared sensor, a low resolution camera, an infrared LED and a GSM modem. When the infrared detector and the camera detect the motion, the alarm will be triggered. When the alarm is triggered, the camera will record a few photos and then send the multimedia message to the receiver's preset phone using a GSM modem. Among them, infrared LED has night vision function; On-the-spot monitoring function. It can clearly hear the voice of the scene, people walk, dig, and other action sounds. Mainframe power break prompt function. If alternating current outages or has electricity, the message will be sent to user to inform. It can be at home to defend, go out to defend, withdraw or emergency alarm through wireless remote control, which is convenient to use. It can be used for remote routing, withdrawal, monitoring, remote output and host function setting through having calls or sending text messages in different places.

\section{Conclusion}

At present, intelligent multi-function door technology is booming, there are many categories of products flooded in the market, belonging to emerging industries. It can be predicted that in the near future, with the breakthrough of technology and the expansion of the market, the intelligent multi-function door will gradually replace the traditional door, making residents' residence more comfortable and environmentally friendly.

\section{Reference}

[1] Haixiang Zhu, Puhua Wen, Yang Pei. The design and implementation of multi-functional smart home system [J]. Innovation and Application of Science and Technology, 2015(07):6-7.

[2] Yuanyi Zhang, Jingquan Zhang, Chunlai Wang. Based on ZigBee's smart home system design[J]. Engineering Technology Research, 2016(08):12-16.

[3] Hongxu Ji, Jibao Li. Intelligent home system based on the Internet of things [J]. Digital Technology Application, 2016(11):74.

[4] Ting Zou. The design of multi-function intelligent home system [J]. Application Technology and Design, 2017(10):73-74. 